\title{
Transfer of the Facultatively Anaerobic Organism Bacteroides corrodens Eiken to a New Genus, Eikenella
}

\author{
F. L. JACKSON and YVONNE E. GOODMAN
}

Department of Medical Bacteriology, University of Alberta, Edmonton, Alberta, Canada

It is proposed that the facultatively anaerobic, gram-negative organism described by Eiken (1958) and named by him Bacteroides corrodens be transferred to a new genus, which the authors name Eikenella. Organisms of this kind form a fairly homogeneous group and differ in important respects from the generally accepted Bacteroides species and from the members of the currently recognized genera of the family Brucellaceae, wherein the genus Eikenella is placed. An up-dated description of the type species, E. corrodens (Eiken) comb. n. (basionym: B. corrodens Eiken 1958), is given. The type strain of this species, previously designated by Henriksen, is Henriksen's strain 333/54-55 (ATCC 23834; NCTC 10596), one of the strains originally studied by Eiken.

Certain gram-negative "corroding bacilli" have been subjected to detailed investigation during the past few years $(2,5,6)$, and they appear not to fit easily into any of the currently recognized genera. Organisms of this type were examined by Eiken (1), who placed them in the genus Bacteroides, under the name $B$. corrodens, because most of the strains with which he worked either were anaerobic or adapted to aerobic growth only after a number of subcultures. More recent work (6) has shown that the aerobic grow th of these organisms may be dependent on the constitution of the medium, particularly on the hemin content. Moreover, the moles per cent guanine plus cytosine (GC) content of the deoxyribonucleic acid (DNA) of all strains tested lies in the 57 to 58 range, and this is widely different from the value for the accepted Bacteroides species. Prévot (9), who does not accept the validity of the generic name Bacteroides but uses the name Ristella, states that members of the genus have an adenine plus thymine to GC ratio of 1.4 , which is equivalent to a GC content of $41 \%$. Similar values for members of the genus Bacteroides have been quoted by Hill (4). Strains of B. fragilis $(42.2 \%)$ and B. melaninogenicus $(41 \%)$ that we have examined gave values close to this figure.

The purpose of this paper is to designate a new genus to contain the facultatively anaerobic strains of the type to which Eiken gave the name $B$. corrodens.

\section{MATERIALS AND METHODS}

Bacterial strains. See Table 1.

Methods. The methods used in this study have been described previously $(5,6)$.

\section{RESULTS AND DISCUSSION}

The facultatively anaerobic "corroding bacilli" of the type studied by Eiken are uniformly oxidase-positive, and this is another divergence from the usually accepted criteria for membership in the genus Bacteroides. There is a wide range of oxygen tolerance among Bacteroides species, and some strains of $B$. fragilis will tolerate up to $8 \%$ oxygen (8). Over the past 5 years, we have isolated more than 200 strains of the Eiken type of corroding bacillus, and no strain has failed to grow under aerobic conditions on first subculture. About half of the strains were detected first on anaerobic plates, and this was sometimes due to suppression of other bacteria which might have obscured the presence of the corroding bacilli on aerobic plates. It is possible too that organisms which have been growing in the body in a lesion where the oxidation-reduction potential is low might take some hours to adapt to aerobic conditions.

Members of the genus Brucella have a GC content of 55 to $58 \%$ (5), but the brucellae differ in important respects from the faculta- 
TABLE 1

\begin{tabular}{|c|c|}
\hline Strain no. ${ }^{a}$ & Source \\
\hline $\begin{array}{c}333 / 54-55(\text { ATCC } \\
\text { NCTC } 10596)\end{array}$ & $\begin{array}{l}\text { Isolated by } \\
\text { Eiken from } \\
\text { sputum }\end{array}$ \\
\hline A124/68 & Liver abscess \\
\hline $\begin{array}{l}\text { MU 53-68 } \\
\quad \text { (NCTC 10647) }\end{array}$ & Sputum \\
\hline R39-71 & Brain abscess \\
\hline MU 10947-64 & Ear \\
\hline MU 639-65 & Abdominal drain site \\
\hline MU $13835-65$ & Peritonsillar abscess \\
\hline MU 19494-65 & Sputum \\
\hline MU 148-66 & Hand \\
\hline MU 5655-66 & Ear \\
\hline MU 8126-66 & Abdominal cavity \\
\hline MU 17272-66 & Finger \\
\hline MU 4353-67 & Abdomen \\
\hline MU 8407-67 & Sputum \\
\hline MU $11350-67$ & Sputum \\
\hline MU 15991-67 & Mouth lesion \\
\hline MU 16367-67 & Sputum \\
\hline MU 17550-67 & Sputum \\
\hline MU 18088-67 & Sputum \\
\hline MU 18548-67 & Sputum \\
\hline MU 24842-67 & Sputum \\
\hline MU 13332-68 & Finger \\
\hline MU 13478-68 & Sputum \\
\hline MU $14030-68$ & Drainage-chest tube \\
\hline
\end{tabular}

${ }^{a}$ Strain 333/54-55 was from the National Collection of Type Cultures (NCTC), London, England. Strain A124/68 was from A. J. Johnston, Victoria, Canada. Strain R39-71 was from the Provincial Laboratory of Public Health, Edmonton, Canada. All MU strains were from the University of Alberta Hospital, Edmonton, Canada. ATCC = American Type Culture Collection, Rockville, Md.

tively anaerobic corroding bacilli, particularly in producing catalase and urease. We would agree with Henriksen (2) that the facultatively anaerobic corroding organisms might reasonably be regarded as falling within the family Brucellaceae, and it seems clear that they could not be placed in the genus Brucella.

Superficially the organisms resemble Haemophilus species, and cultures have an odor resembling that produced by cultures of the genera Pasteurella and Haemophilus. The moles $\%$ GC values of all authentic Haemophilus strains that have been examined are in the 37 to $42 \%$ range (5). The GC contents of pasteurellas are closely similar to those of Bacteroides species, 39.5 to $42 \%$ (6), whereas those of yersiniae are significantly higher and are close to $48 \%$ (Jackson et al., unpublished data).

Antigenic studies (6) have shown that the facultatively anaerobic corroding organisms are closely interrelated but that quantitative differences occur between strains. Certain stains may lack one or two of the four major antigenic components detected by immunodiffusion studies (P.C. Wong and F.L. Jackson, Bacteriol. Proc., p. 54, 1971).

Strains of anaerobic, gram-negative bacilli which produce pitting of the surface of agar media have also been called $B$. corrodens. Strictly anaerobic pitters which we have examined have always shown important differences from the facultatively anaerobic strains, the GC contents of the former being 28 to $30 \%$. These strains produce urease, are weakly proteolytic, and attack starch. They are antigenically unrelated to the facultatively anaerobic strains (6). We have not encountered any anaerobic (i.e., oxygen-intolerant) strains which could be regarded as anaerobic biotypes of the facultatively anaerobic organisms. Anaerobic strains of the kind we have studied should not be called B. corrodens, as their low DNA GC content differs widely from that of generally accepted Bacteroides species.

It seems probable that the facultatively anaerobic corroding bacilli are the same as the organisms referred to as subgroup HB-1 by King (7). Strain A 124/68 (nonpitting), which was isolated from a liver abscess by Anne Johnston, Royal Jubilee Hospital, Victoria, British Columbia, was sent by her to the National Center for Disease Control, Atlanta, where it was identified as HB-1.

On the basis of findings reported in the publications quoted above, and taking into account our own experience with the facultatively anaerobic, corroding bacilli of the Eiken type, we propose the recognition of a new genus, Eikenella (M.L. dim. ending-ella; M.L. fem. n. Eikenella named for M. Eiken, who first named the type species of this genus), which is placed in the family Brucellaceae (Breed et al.) and which is described as follows:

Gram-negative rods, 0.3 to 0.4 by 1.5 to 4 $\mu \mathrm{m}$, with a characteristically regular morphology.

Filaments occasionally formed.

Unbranched.

Nonsporing.

Nonmotile.

Catalase negative.

Oxidase positive.

Aerobic and facultatively anaerobic. 
TABLE 2. Distinguishing features of Eikenella and superficially similar genera

\begin{tabular}{|c|c|c|}
\hline Genus & DNA: moles $\% \mathrm{GC}^{a}$ & Other useful distinguishing features \\
\hline Eikenella & $57-58(5,6)$ & $\begin{array}{l}\text { Oxidase positive, catalase negative, urease } \\
\text { negative, indole negative, nonfermentative, } \\
\text { facultatively anaerobic }\end{array}$ \\
\hline Actinobacillus & $38-43(5)$ & Fermentative \\
\hline Bordetella & $64-68(5)$ & Usually catalase positive \\
\hline Brucella & $55-58(5)$ & Catalase positive, urease positive \\
\hline Cardiobacterium & $61.7(5)$ & Indole positive, fermentative \\
\hline Haemophilus & $37-42(5)$ & Glucose usually attacked, with production of acid. \\
\hline Moraxella & $38-45(5)$ & Catalase positive, proteoly tic \\
\hline Pasteurella & $39-42^{b}$ & Catalase positive, fermentative \\
\hline Yersinia & $46-50^{b}$ & Catalase positive, fermentative \\
\hline Bacteroides & $38-42(6,9)$ & Oxidase negative, characteristically anaerobic \\
\hline
\end{tabular}

$a$ Numbers in parentheses refer to literature cited. Moles $\% \mathrm{GC}=$ guanine plus cytosine.

$b$ Jackson et al. (6) and unpublished data.

Hemin usually required for aerobic growth. No detectable acid production from carbohydrates.

The GC content is 57 to 58 moles \%.

Probably normal inhabitants of the mouth and upper respiratory tract of man.

Type species: Eikenella corrodens (Eiken) comb. $\mathrm{n}$.

Basionym: Bacteroides corrodens Eiken 1958.

Type strain: Henriksen's strain 333/54-55 (ATCC 23834; NCTC 10596). This strain is one of those originally studied by Eiken (1); it was designated as the type by Henriksen $(2,3)$.

Table 2 lists important features which assist in differentiation of Eikenella from other superficially similar genera.

Description of E. corrodens. E. corrodens are gram-negative rods, 0.3 to 0.4 by 1.5 to $4 \mu \mathrm{m}$. The morphology is regular; the organisms appear stick-like, straight, with rounded ends. They are unbranched, nonmotile, nonflagellated, and nonsporing.

Colonies on blood-agar at $24 \mathrm{hr}$ were $0.5 \mathrm{~mm}$; at $48 \mathrm{hr}, 0.5$ to $1.0 \mathrm{~mm}$ in diameter. Corroding strains appear as if in a shallow pit in the agar, and the colony appears rough to the naked eye. The edge is circular or irregular. Nonpitting strains may be encountered and give domeshaped colonies 0.5 to $1.0 \mathrm{~mm}$ in diameter.
Colonies are grey, translucent, and nonhemolytic; they sometimes have a small, greenish zone around the edge after $48 \mathrm{hr}$ or more of incubation. The odor of cultures resembles that of organisms in the genera Pasteurella and Haemophilus.

Growth in fluid media is usually poor, but it may be improved by the addition of cholesterol $(10 \mu \mathrm{g} / \mathrm{ml}$; reference 3$)$ or $3 \%$ sheep serum. Growth in a semisolid medium with $0.1 \%$ agar is better than that in a fluid medium.

Growth is favored by 5 to $10 \% \mathrm{CO}_{2}$, especially on primary isolation and often on subsequent transfer for many subcultures.

This organism typically requires hemin for aerobic growth. Best growth is obtained with hemin concentrations of 5 to $25 \mu \mathrm{g}$ per $\mathrm{ml}$ of medium. Non-hemin-requiring variants can be selected in vitro.

Nonfermentative in that acid and gas are not produced from glucose or from a wide range of other carbohydrates.

Oxidase positive, catalase negative. Nitrate reduced to nitrite.

Urease negative, gelatinase negative, indole negative.

Lysine decarboxylase positive.

Typical strains are aerobic and facultatively anaerobic. The existence of obligately anaerobic biotypes has not been rigorously proven. 


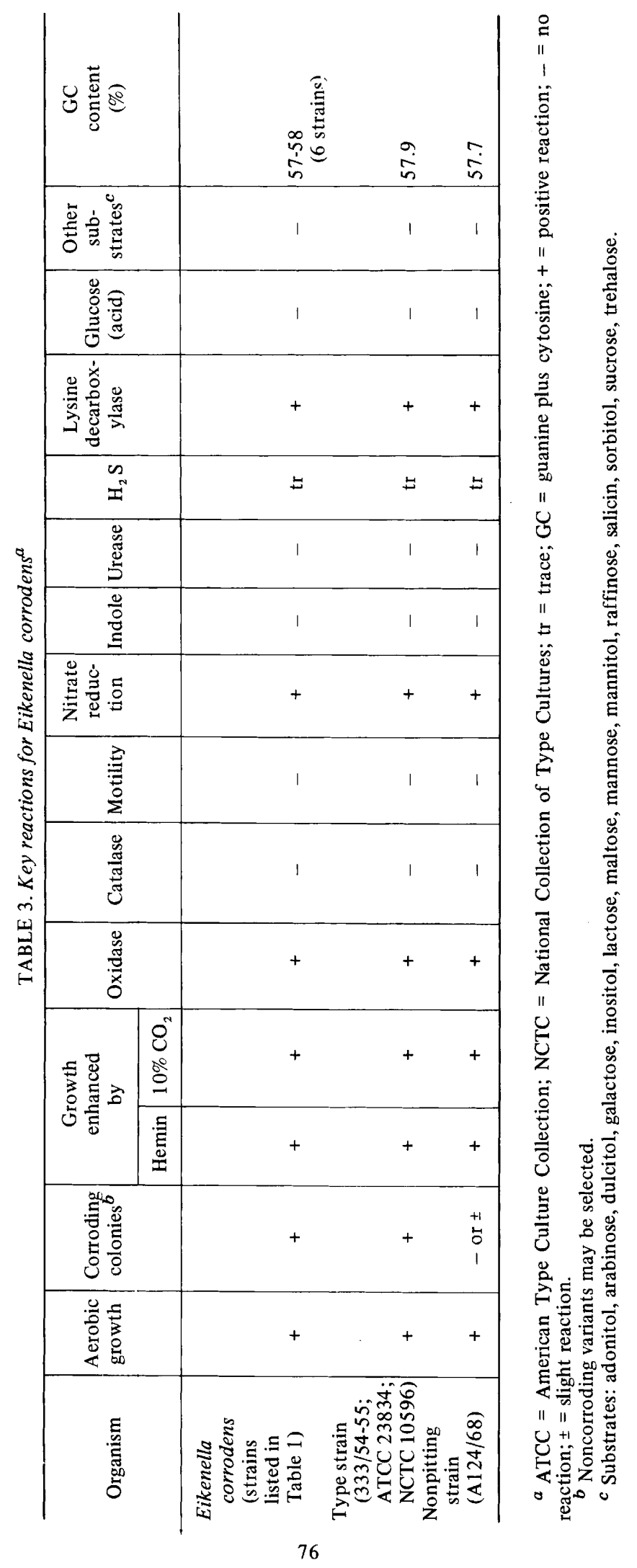


Growth under anaerobic conditions is favored by 5 to $10 \% \mathrm{CO}_{2}$. Growth under anaerobic conditions is improved by the addition of $0.1 \%$ $\mathrm{KNO}_{3}$ to the medium, and it is better with $1 \%$ oxygen than under strictly anaerobic conditions.

The moles \% GC of the DNA is 57 to 58 .

In electron micrographs of phosphotungstate negatively stained organisms, the cell surface appears finely convoluted (a "cerebral" appearance).

Probably a normal inhabitant of the mouth and upper respiratory tract of man. The evidence available suggests that this organism may be an opportunistic pathogen and may sometimes occur alone in infections. Usually occurs as one member of a complex flora in clinical specimens. It has been isolated from sputum, brain abscess, liver abscess, osteomyelitis, and infected wounds. Nonpitting variants may be isolated directly from clinical specimens.

Antigenic structure: all strains examined share some antigens, but quantitative differences occur, and one or more antigens may be lacking. Immunodiffusion studies indicate that there are four major antigens.

Table 3 presents a summary of the key features of strains studied in detail.

In defining the genus, we have incorporated features which are based on reactions obtained when the methods used were as described by Hill, Snell, and Lapage (5), and Jackson et al.
(6). It cannot be too strongly stressed that for comparison of data obtained by two or more laboratories, standardization of methods is essential.

\section{LITERATURE CITED}

1. Eiken, M. 1958. Studies on an anaerobic, rodshaped Gram negative microorganism: Bacteroides corrodens N. sp. Acta Pathol. Microbiol. Scand. 43: 404-416.

2. Henricksen, S. D. 1969. Corroding bacteria from the respiratory tract. II. Bacteroides corrodens. Acta Pathol. Microbiol. Scand. 75:91-96.

3. Henriksen, S. D. 1969. Designation of the type strain of Bacteroides corrodens Eiken 1958. Int. J. Syst. Bacteriol. 19:165-166.

4. Hill, L. R. 1966. An index to deoxyribonucleic acid base compositions of bacterial species. J. Gen. Microbiol. 44:419-437.

5. Hill, L. R., J. J. S. Snell, and S. P. Lapage. 1970 Identification and characterization of Bacteroides corrodens. J. Med. Microbiol. 3:483-491.

6. Jackson, F. L., Y. E. Goodman, F. R. Bel, P. C. Wong, and R. L. S. Whitehouse. 1971. Taxonomic status of facultative and strictly anaerobic "corroding bacilli" that have been classified as Bacteroides corrodens. J. Med. Microbiol. 4:171-184.

7. King, E. O., and H. W. Tatum. 1962. Actinobacillus actinomycetemcomitans and Hemophilus aphrophilus. J. Infect. Dis. 111:85-94.

8. Loesche, W. J. 1969. Oxygen sensitivity of various anaerobic bacteria. Appl. Microbiol. 18:723-727.

9. Prévot, A.-R., A. Turpin, and P. Kaiser. 1967. Les bactéries anaérobies, p. 200. Dunod, Paris. 